05

\title{
Жесткая фокусировка ультракоротких лазерных импульсов в объем ZnSe
}

\author{
( Ю.С. Гулина ${ }^{1,2}$, С.И. Кудряшов ${ }^{2}$, Н.А. Смирнов ${ }^{2}$, Е.В. Кузьмин ${ }^{2}$ \\ ${ }^{1}$ Московский государственный технический университет им. Н.Э. Баумана, \\ 105005 Москва, Россия \\ ${ }^{2}$ Физический институт им. П.Н. Лебедева, \\ 119991 Москва, Россия \\ e-mail: julia-sg@yandex.ru
}

Поступила в редакцию 20.12.2021 г.

В окончательной редакции 20.12.2021 г.

Принята к публикации 30.12.2021 г.

Исследован процесс жесткой фокусировки ультракоротких лазерных импульсов в объем ZnSe. Показано, что при фокусировке лазерного гауссова пучка высокоапертурными объективами внутрь прозрачной среды возникают аберрационные искажения, приводящие к увеличению фокального пятна. Приведены результаты экспериментальных исследований по абляции передней и тыльной поверхностей плоскопараллельной пластинки толщиной $4 \mathrm{~mm}$, выполненной из материала $\mathrm{ZnSe}$, лазерными импульсами длительностью $0.3,1$, $10 \mathrm{ps}$ и длиной волны $1030 \mathrm{~nm}$, сфокусированными микрообъективом $(N A=0.55)$. Показано, что при низких энергиях импульсов фокусировку можно рассматривать в линейном режиме, в котором размер фокального пятна обусловлен аберрационными искажениями.

Ключевые слова: прямая лазерная запись, фемтосекундные лазерные импульсы, жесткая фокусировка, абляция, аберрации.

DOI: 10.21883/OS.2022.04.52261.45-21

\section{Введение}

В настоящее время активно изучаются возможности использования методов лазерной обработки с помощью ультракоротких импульсов для изготовления сложных структур и приборов [1-4]. Прямая лазерная запись может быть использована для создания сложных трехмерных пространственных структур с субмикронным разрешением внутри прозрачных сред. Такие структуры нужны для разработки информационнотелекоммуникационных и биоинженерных приложений следующего поколения: оптической памяти [5], микрооптических компонент [6], решеток показателя преломления [7], оптических волноводов [8] и т. п. Высокая точность, скорость обработки и универсальность делают лазерную обработку ультракороткими импульсами важным инструментом для производства. Для обеспечения максимальной эффективности обработки используется жесткая фокусировка лазерного излучения в объем модифицируемой прозрачной среды, позволяющая получить необходимую надпороговую величину интенсивности лазерного излучения и локального энерговклада в области фокусировки [9].

Лазерная энергия в основном поглощается в фокальном пятне из-за нелинейных особенностей взаимодействия лазера с диэлектриком [10,11]. Поглощение энергии приводит к модификации материала, и размер областей абляции, по-видимому, является одним из наиболее важных параметров, которые влияют на эффективность, а также на пространственное разрешение микрообработ- ки. Для пороговых процессов модификации, таких как абляция, радиус модифицируемой области зависит от порогового потока $f_{t h}$, энергии импульса $E$ и радиуса фокального лазерного пятна $w_{0}$, определяемого [12] по уровню энергии $1 / e$ :

$$
R_{a b l}^{2}\left(w_{0}^{2}\right)=w_{0}^{2} \ln \left(\frac{E}{F_{t h} \pi w_{0}^{2}}\right) .
$$

Чаще всего физические эффекты, используемые для создания контролируемого повреждения внутри обрабатываемых материалов, требуют фокусировки лазерного пучка в небольшое пятно, размер которого обычно составляет несколько микрометров. Это достигается за счет использования высокоапертурных объективов или специально разработанных асферических линз, которые скоррегированы для конкретной рабочей плоскости в воздухе или внутри прозрачной среды, например, для задней поверхности покровного стекла. Поэтому размер пятна в заранее определенной рабочей плоскости определяется волновой природой света, т.е. является дифракционно ограниченным.

При фокусировке гауссова пучка в воздухе его радиус на заданном расстоянии $z$ от объектива может быть определен как

$$
w(z)=w_{0} \sqrt{1+\left(\frac{z-f^{\prime}}{z_{R}}\right)^{2}},
$$

где $z=f^{\prime}-$ расстояние до геометрического фокуса, а $z_{R}=\frac{\pi w_{0}^{2}}{\lambda}-$ длина Рэлея, $\lambda-$ длина волны лазерного 
излучения, $w_{0}=\frac{\lambda f^{\prime}}{\pi w(0)}-$ радиус фокального пятна, который может быть также выражен через числовую апертуру $N A$ фокусирующей оптики:

$$
w_{0}=\frac{\lambda}{\pi} \frac{\sqrt{1-N A^{2}}}{N A}
$$

$w(0)$ - радиус лазерного пучка перед фокусирующей системой.

При фокусировке гауссова пучка внутрь прозрачного материала в параксиальном приближении происходит смещение положения фокальной плоскости. При этом размер сфокусированного пятна аналогичен размеру пятна в воздухе, а расстояние Рэлея увеличивается пропорционально показателю преломления материала:

$$
z_{R(\text { mat })}=n \frac{\pi w_{0}^{2}}{\lambda}=n z_{R(\text { air })} .
$$

На практике, когда гауссов пучок фокусируется на разных глубинах внутри прозрачной среды, возникает положительная сферическая аберрация, влияющая на размер сфокусированного пятна. При числовых апертурах $N A>0.5$ размер фокального пятна может в несколько раз превышать дифракционно ограниченное пятно [13]. Это неизбежно влияет на концентрацию лазерной энергии в сфокусированном пятне и снижает пространственное разрешение лазерной модификации. Для минимизации влияния аберраций на размер фокального пятна можно использовать оптику с более низкими апертурами, однако при этом в случае значительных энергий импульсов фокусировка будет искажена нелинейными эффектами: самофокусировкой Керра, образованием плазмы, филаментацией.

Положительная сферическая аберрация, возникающая при прохождении сфокусированным пучком границы раздела воздух-материал, вызывает смещение положения плоскости фокусировки относительно параксиального положения, причем для разных зон пучка это смещение будет различно - от максимального для краевой зоны до минимального для центральной зоны (рис. 1).

Значение максимального продольного смещения плоскости фокусировки $\Delta s_{2}^{\prime}$ можно оценить по формуле

$$
\Delta s_{2}^{\prime}=z_{2}^{\prime}-z_{0}^{\prime}=\frac{n_{2}}{n_{1}} \sqrt{h^{2}\left(1-\frac{n_{1}^{2}}{n_{2}^{2}}\right)+\frac{z_{0}^{\prime 2}}{n_{2}}}-z_{0}^{\prime},
$$

где $z_{2}^{\prime}$ - расстояние от границы раздела до плоскости фокусировки крайних лучей пучка, $z_{0}^{\prime}-$ расстояние от границы раздела до параксиальной фокальной плоскости, $n_{1}$ и $n_{2}$ - показатели преломления сред, $h-$ радиус фокусируемого пучка на границе раздела сред.

Продольное смещение плоскостей фокусировки (3) приводит к появлению в параксиальной фокальной плоскости поперечной сферической аберрации $\Delta y_{s p h}^{\prime}$, величину которой можно определить по формуле

$$
\Delta y_{s p h}^{\prime}=\Delta s_{2}^{\prime} \operatorname{tg}\left(\frac{h}{z_{2}^{\prime}}\right) \text {. }
$$

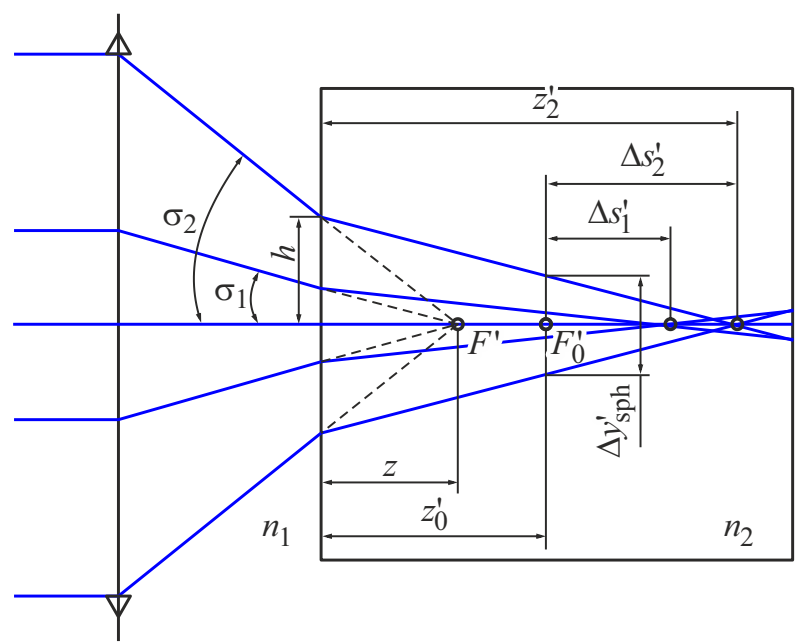

Рис. 1. Расчетная схема для определения сферической аберрации.

Чем больше числовая апертура фокусирующей оптики или чем глубже пучок фокусируется внутри прозрачной среды, тем больше сферическая аберрация и, следовательно, сильнее рассеяние световой энергии в фокальной области. Размер фокального пятна с учетом аберрационных искажений (4) будет определяться как

$$
w_{\text {aber } 0}=w_{0}+\Delta y_{s p h}^{\prime} .
$$

Таким образом, повышение апертуры, с одной стороны, приводит к уменьшению параксиального пятна, а с другой - к аберрационному увеличению, поэтому необходимо определять оптимальную числовую апертуру, обеспечивающую минимальной размер сфокусированного пятна или использовать адаптивную коррекцию сферической аберрации. Зависимости параксиального и аберрационного размеров пятна, а также сферической аберрации от эффективной числовой апертуры приведены на рис. 2. Эффективная числовая апертура определяется через размер фокусируемого пятна на границе раздела сред и глубину фокусировки:

$$
N A_{e f f}=\sin \left(\operatorname{arctg}\left(\frac{h}{z_{0}^{\prime}}\right)\right) .
$$

Таким образом, размер области абляции на заданном расстоянии $z$ от объектива с учетом формул (1), (2) и (5) может быть определен по предложенной формуле

$$
R_{\text {ablaberr }}^{2}(z)=w_{\text {aberr }}^{2}(z) \ln \left(\frac{E}{F_{t h} \pi w_{\text {aberr }}^{2}(z)}\right),
$$

где размер фокусируемого пучка на заданном расстоянии $z$ определяется с учетом аберрационных искажений:

$$
w_{\text {aberr }}(z)=w_{\text {aberr }} 0 \sqrt{1+\left(\frac{z-f^{\prime}}{z_{R(\text { mat }}}\right)^{2}} .
$$




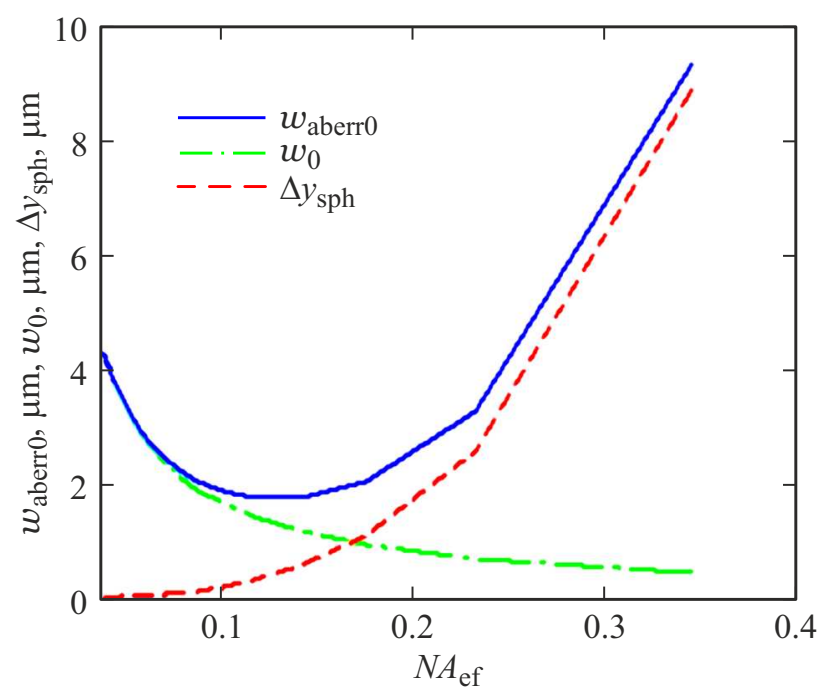

Рис. 2. Зависимость параксиального и аберрационного размеров пятна, а также сферической аберрации от эффективной числовой апертуры.

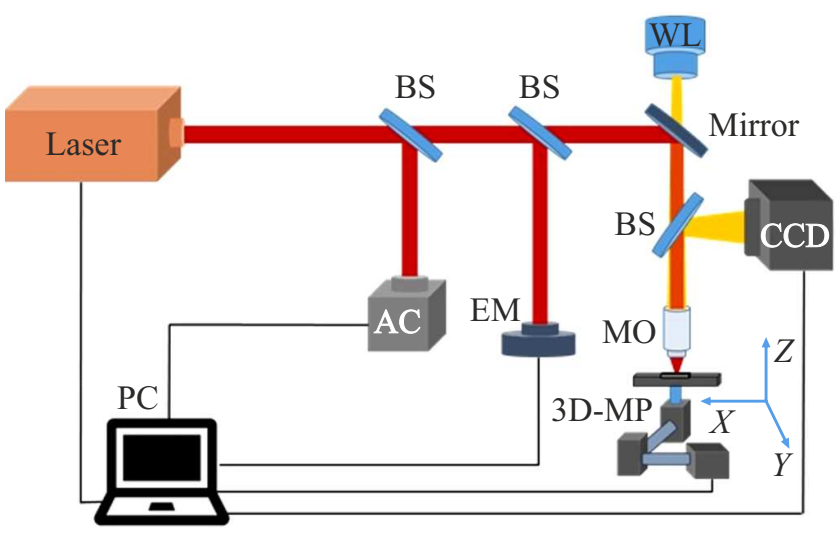

Pис. 3. Схема рабочей станции для фс/пс-лазерной абляции: BS - светоделитель, ЕМ - счетчик энергии, АC - автокоррелятор, $\mathrm{MO}$ - микрообъектив, $\mathrm{WL}$ - источник белого света, РC - компьютер со специальным программным обеспечением для управления лазером, камерой, системой позиционирования, CCD - камера для визуализации поверхности во время сканирования.

\section{Экспериментальная часть}

Для оценки влияния аберрационных искажений на жесткую фокусировку гауссова пучка в объем прозрачных диэлектриков был проведен ряд экспериментальных исследований, в ходе которых производилась абляция передней и тыльной сторон образца в виде полированной с двух сторон плоскопараллельной пластинки толщиной $4 \mathrm{~mm}$, выполненной из материала $\mathrm{ZnSe}$. Схема экспериментальной установки, используемой для лазерной абляции, приведена на рис. 3.

В качестве источника лазерного излучения был использован волоконный лазер Satsuma (Amplitude
Systemes) с активной средой на ионах $\mathrm{Yb}^{+3} \mathrm{c}$ длиной волны $1030 \mathrm{~nm}$ и линейной поляризацией. Длительность импульсов, регулируемая с помощью встроенного компрессора, составляла $0.3,1$ и $10 \mathrm{ps,} \mathrm{а} \mathrm{частота} \mathrm{следова-}$ ния импульсов $-0.2 \mathrm{kHz}$. Образец был закреплён на трёхкоординатной подвижной платформе, позволившей производить двухкоординатное сканирование $Z-Y-$ изменение глубины фокусировки совместно с перемещением образца, что обеспечило последовательную фокусировку (в объёме образца - на поверхности образца над поверхностью образца). При этом при облучении тыльной стороны излучение заводилось непосредственно через переднюю сторону образца. Для фокусировки был использован микрообъектив с $N A=0.55$ и фокусным расстоянием $f=5 \mathrm{~mm}$. Абляционные кратеры получены при энергиях в импульсе от 0.07 до $0.85 \mu \mathrm{J}$ и динамической фокусировке лазерного пучка. Энергия лазерного излучения измерялась с помощью измерителя энергии Ophir PD10-C.

\section{Результаты и их обсуждение}

Абляция передней поверхности была выполнена для проведения калибровки и определения порогов модификации материала. Изображения экспериментальных областей абляции, полученные на сканирующем электронном микроскопе (СЭМ) Tescan Vega Compact, приведены на рис. 4.

На основе измерения поперечных размеров этих областей были построены зависимости квадрата радиуса абляционного кратера $R_{a b l}^{2}$ от логарифма энергии $\ln E$ для различных длительностей импульса (рис. 5). Наклон полученных зависимостей [14] определяет характерные размеры фокальных пятен $w_{a b l}=(\text { Slope })^{1 / 2}$, которые близки к теоретически рассчитанным значениям фокального пятна $w_{0}=1.2 \mu \mathrm{m}$ для лазерного пучка с радиусом по уровню $1 / e w(0)=0.7 \mathrm{~mm}$. Значения порогов модификации, рассчитанные через пороговые энергии для различных длительностей импульсов $F_{t h}=E_{t h} /\left(\pi w_{a b l}^{2}\right)$, приведены на рис. 5.

Теоретически определенные на основе предложенной формулы (6) формы областей абляции хорошо согласуются с видом экспериментально полученных ожогов на передней поверхности образца (рис. 6, $a$ ): для малых энергий импульсов форма ожогов гладкая, а для высоких наблюдается характерный изгиб в области плоскости фокусировки - гантелеобразная форма ожогов.

Изображения областей абляции на тыльной поверхности образца, полученные на СЭМ Tescan Vega Compact, приведены на рис. 7.

Зависимости квадрата ширины абляционного кратера $R_{a b l}^{2}$ от логарифма энергии $\ln E$ для различных длительностей импульса, полученные на основе измерения областей абляции на тыльной поверхности образца, а также характерные размеры фокальных пятен и значения порогов модификации представлены на рис. 8. В об- 

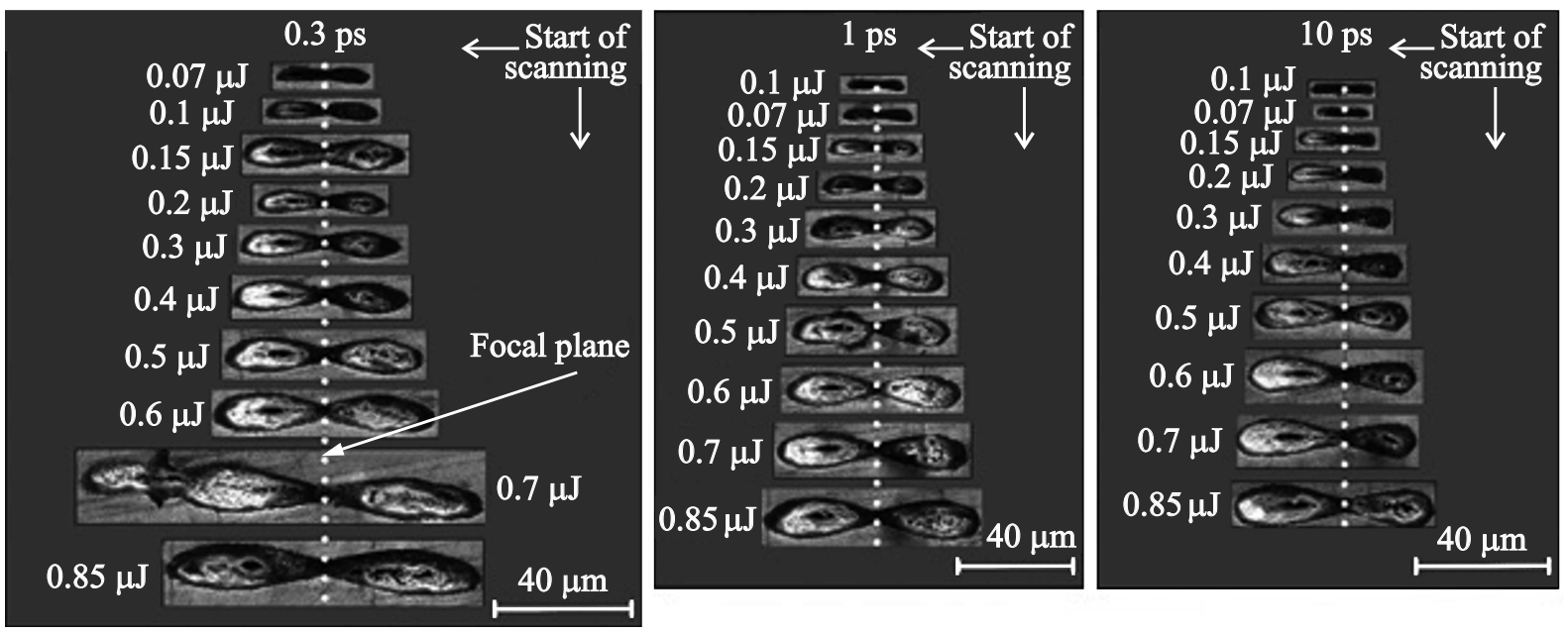

Рис. 4. Абляционные области на передней поверхности образца.

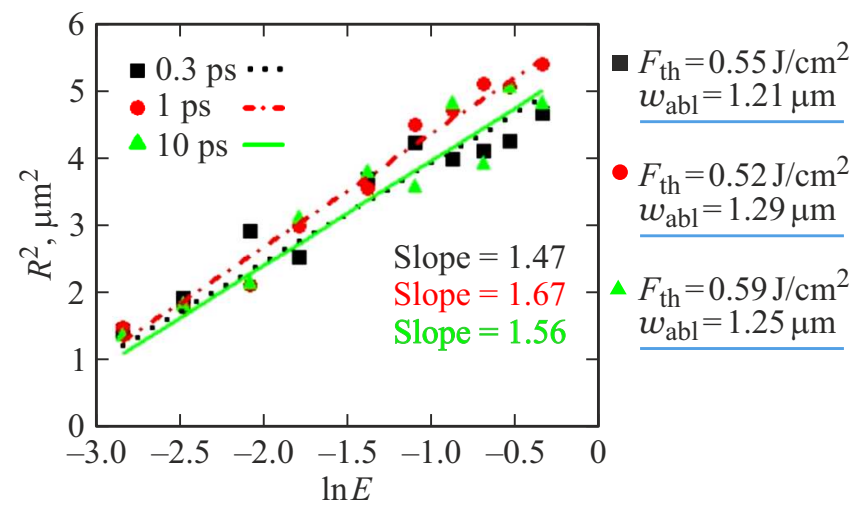

Рис. 5. Зависимость квадрата радиуса области абляции на передней поверхности образца от логарифма энергии импульса.

ласти низких энергий импульсов характерные размеры, полученные из зависимостей $R_{a b l}^{2}-\ln E$, согласуются с теоретически рассчитанным размером фокального пятна при учете аберрационного размытия $w_{\text {aberr }}=1.8 \mu \mathrm{m}$.

Форма зависимостей квадрата радиуса абляции от расстояния, рассчитанных на основе формулы (6), учитывающей аберрациию, для области низких энергий также согласуется с видом экспериментальных ожогов - они имеют овальную форму, в то время как без учета аберрационных искажений теоретическая форма имеет гантелеобразный вид (рис. 6, $b$ ). Однако в области высоких энергий импульсов наблюдается несоответствие теоретических и экспериментально полученных характерных размеров фокальных пятен даже при учете аберрационных искажений - теоретически рассчитанные значения занижены относительно экспериментальных. Повидимому, это связано с нелинейным характером фокусировки, обусловленным наличием экранирующей плазмы, самофокусировки и филаментации, возникающими при больших энергиях. Стоит отметить, что наличие
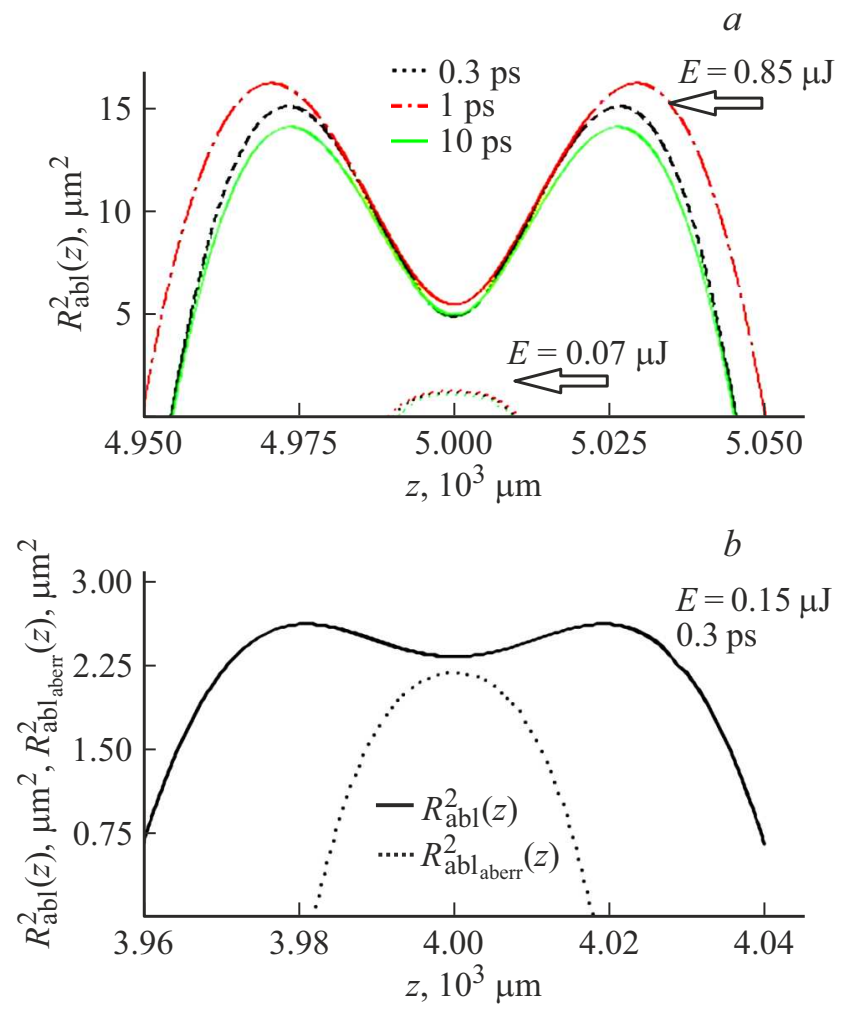

Рис. 6. Зависимости квадрата радиуса области абляции на передней поверхности от расстояния от объектива $(a)$ и квадрата радиуса области абляции на тыльной поверхности от расстояния от границы раздела $(b)$.

нелинейных эффектов при жесткой фокусировке в объем прозрачных материалов обусловлено уменьшением эффективной фокусирующей апертуры на границе раздела воздух-среда пропорционально показателю преломления среды. Таким образом, даже при использовании объективов с высокими апертурами для фокусировки в объем 

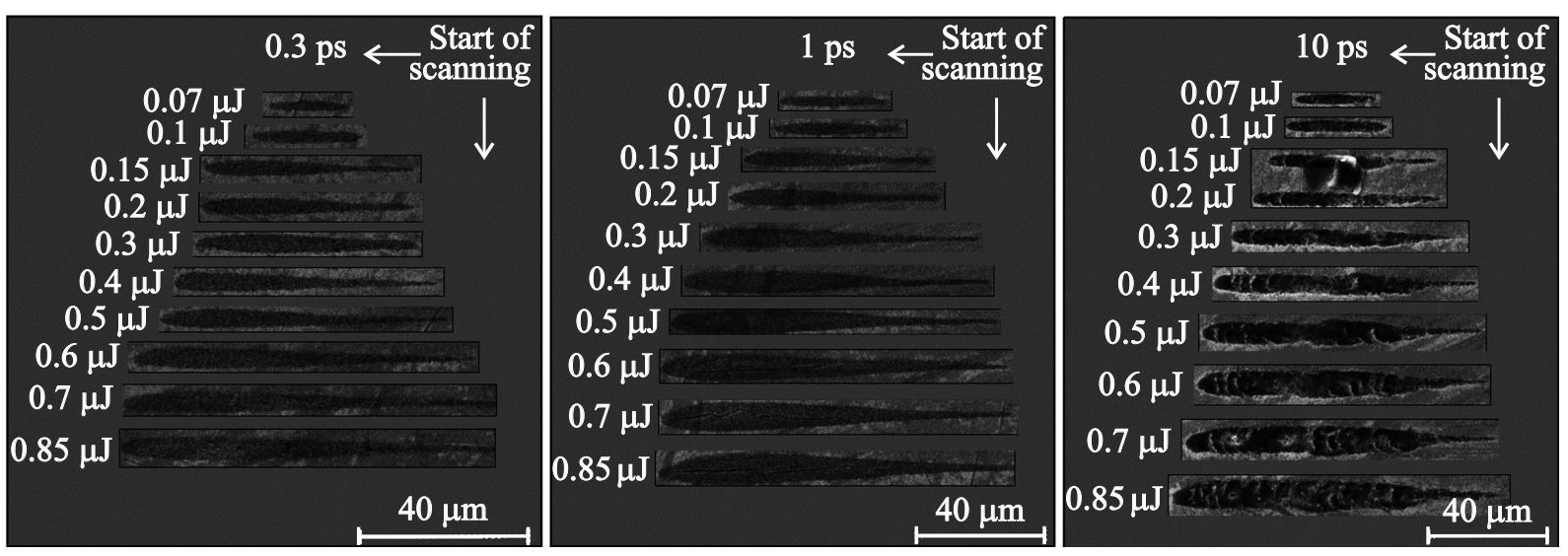

Рис. 7. Абляционные области на тыльной поверхности образца.

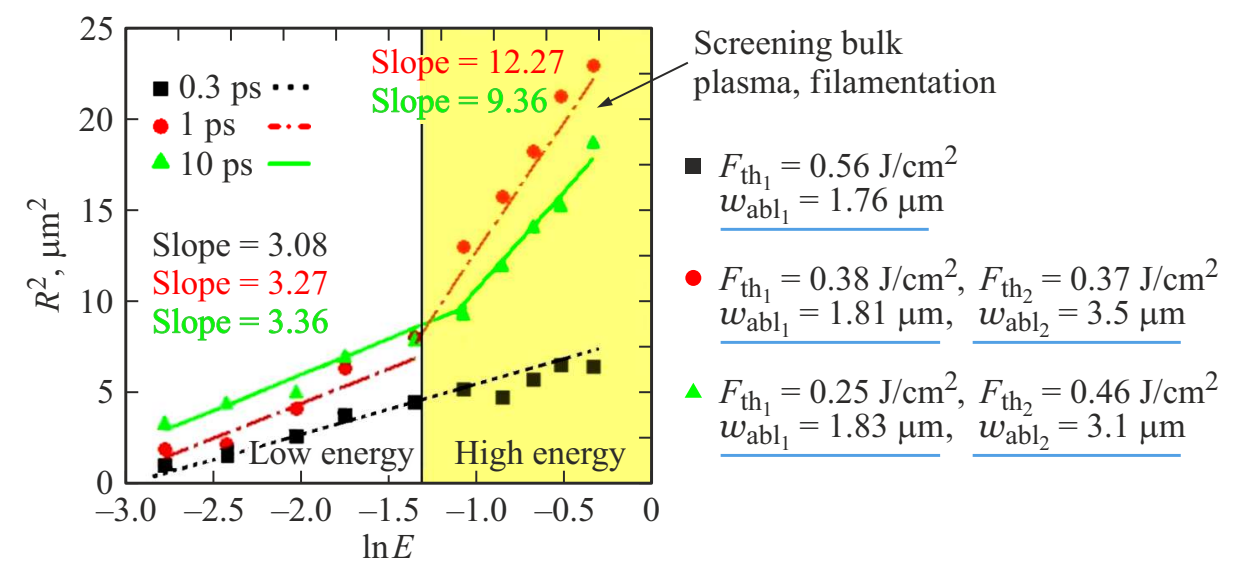

Рис. 8. Зависимость квадрата радиуса области абляции на тыльной поверхности образца от логарифма энергии импульса.

прозрачных сред с большим показателем преломления эффективная рабочая апертура понижается, поэтому не происходит ослабления нелинейных эффектов.

\section{Заключение}

Исследован процесс жесткой фокусировки ультракоротких лазерных импульсов в объем ZnSe. Показано, что при анализе размера фокального пятна необходимо учитывать сферическую аберрацию, возникающую из-за искажения волнового фронта сфокусированного гауссова пучка на границе раздела воздух-материал. Отмечено, что в области низких энергий импульсов наблюдается хорошее соответствие значений характерных радиусов абляции, рассчитанных с учетом аберрационных искажений, и экспериментально полученных значений, что говорит о линейном характере фокусировки, в котором размер фокального пятна обусловлен наличием сферической аберрации. В области высоких энергий импульсов при жесткой фокусировке ультракоротких лазерных импульсов в объем $\mathrm{ZnSe}$, а также других прозрачных материалов с высоким показателем преломления фоку- сировка идет в нелинейном режиме из-за уменьшения эффективной рабочей апертуры.

\section{Финансирование работы}

Авторы признательны Российскому научному фонду за финансовую поддержку данных исследований в рамках проекта 21-79-30063.

\section{Конфликт интересов}

Авторы заявляют, что у них нет конфликта интересов.

\section{Список литературы}

[1] L. Cerami, E. Mazur, S. Nolte, C. B. Schaffer. Ultrafast nonlinear optics (Springer, Heidelberg, 2013), p. 287-321. DOI: 10.1007/978-3-319-00017-6_12

[2] K.C. Phillips, H.H. Gandhi, E. Mazur, S.K. Sundaram. Advances in Optics and Photonics, 7 (4), 684 (2015). DOI: 10.1364/AOP.7.000684

[3] R. R. Gattass, E. Mazur. Nature Photonics, 2 (4), 219 (2008). DOI: $10.1038 /$ nphoton.2008.47 
[4] K. Sugioka, Y. Cheng. Light Sci Appl., 3, e149 (2014). DOI: $10.1038 / 1 \mathrm{sa} .2014 .30$

[5] M.H. Hong et al. Appl. Phys. A, 79, 791 (2004).

DOI: $10.1007 / \mathrm{s} 00339-004-2630-1$

[6] J. Qiu, K. Miura, K. Hirao. Optical Components and Materials, 5350, 1 (2004). DOI: 10.1117/12.537372

[7] Y. Shimotsuma, P.G. Kazansky, J. Qiu, K. Hirao. Phys. Rev. Lett., 91, 247405 (2003). DOI: 10.1103/PhysRevLett.91.247405

[8] F. Chen, J.V. de Aldana. Laser Photonics Rev., 8 (2), 251 (2014). DOI: 10.1002/lpor.201300025

[9] C.B. Schaffer, A. Brodeur, J.F. Garcia, E. Mazur. Optics Lett., 26 (2), 93 (2001). DOI: 10.1364/OL.26.000093

[10] J. Peng, D. Grojo, D.M. Rayner, P.B. Corkum. Appl. Phys. Lett., 102, 161105 (2013). DOI: 10.1063/1.4802820

[11] R.R. Gattass, E. Mazur. Nat. Photonics, 2, 219 (2008). DOI: $10.1038 /$ nphoton.2008.47

[12] S. Kudryashov, P. Danilov, A. Rupasov, S. Khonina, A. Nalimov, A. Ionin, G. Krasin, M. Kovalev. Optical Materials Express, 10 (12), 3291 (2020). DOI: 10.1364/OME.412399

[13] A. Laskin, V. Laskin, A. Ostrun. ICALEO, 2017, M404 (2017). DOI: $10.2351 / 1.5138167$

[14] J.M. Liu. Optics Lett., 7 (5), 196 (1982).

DOI: 10.1364/OL.7.000196 